\title{
Germanica
}

\section{Welterfahrungsspiele: Reisethemen in der Gegenwartslyrik}

Stefan Elit: Explorer le monde : le voyage dans la poésie contemporaine

Stefan Elit: Exploring the World playfully: Journeys as Motifs in Contemporary

Lyrical Poetry

\section{Stefan Elit}

\section{(2) OpenEdition}

\section{Journals}

\section{Édition électronique}

URL : http://journals.openedition.org/germanica/6948

DOI : 10.4000/germanica.6948

ISSN : 2107-0784

\section{Éditeur}

Université de Lille

\section{Édition imprimée}

Date de publication : 26 juin 2019

Pagination : 131-148

ISBN : 978-2-913857-42-1

ISSN : 0984-2632

\section{Référence électronique}

Stefan Elit, "Welterfahrungsspiele: Reisethemen in der Gegenwartslyrik“, Germanica [Online], 64 | 2e trimestre 2019, Online erschienen am: 01 Januar 2021, abgerufen am 23 Februar 2021. URL: http:// journals.openedition.org/germanica/6948 ; DOl: https://doi.org/10.4000/germanica.6948 


\title{
Welterfahrungsspiele: Reisethemen in der Gegenwartslyrik
}

\author{
Stefan Elit \\ Universität Paderborn
}

Deutschsprachige Lyrikerinnen und Lyriker reflektieren seit geraumer Zeit signifikant häufiger reale oder auch nur fiktive Reisen und Aufenthalte in ihren Gedichten und teils sogar in ganzen Gedichtbänden. Die Breite des Interesses belegt etwa ein eigens dem Thema Lyriker(innen) und Reisen gewidmeter Band der Zeitschrift Das Gedicht von 2013, der zahlreiche Gegenwartsautorinnen und -autoren mit einzelnen Gedichten sowie mit kurzen Statements zusammenführt ${ }^{1}$. Um diese Gattungsentwicklung zu erfassen, sind zunächst basale Gattungsfragen, Forschungslagen sowie literarhistorische Hintergründe von Lyrik und Reiseliteratur zu umreißen, um dann drei für sich und im Vergleich besonders interessante Werke exemplarisch zu untersuchen. Fokussiert wird dabei die Zeit nach 1989, d.h. seitdem sich generell die Möglichkeiten des Reisens nach West und Ost (und Nord und Süd) stark erweitert haben, und dabei soll es um die reiselyrisch besonders produktiven jüngsten (zehn) Jahre gehen. Herangezogen werden mit einer losen chronologischen Abfolge Werke von Uwe Kolbe (zugleich der älteste Autor, Geburtsjahrgang 1957), der sich in den 1990er Jahren u.a. Italien und in den 2000er Jahren Bulgarien lyrisch erschlossen hat. Es folgt eine Auseinandersetzung mit Jan Wagner (Jg. 1971), der seit den späten 2000er Jahren diverse

1. — Vgl. Das Gedicht, 21/2013, Bd. 21: Pegasus \& Rosinante. Wenn Poeten reisen. 
globale und regionale Räume in Europa oder Amerika bedichtet hat, sowie eine Werksichtung für Ulrike Almut Sandig (Jg. 1979), die in Gedichtbänden um 2010 reisethematisch besonders aktiv geworden ist ${ }^{2}$.

\section{Entautomatisierung durch Reiselyrik und die Entwicklung von deren literarischer Reihe}

Für die Frage nach dem poetologischen Standort von Gegenwartslyrik zu Reisethemen sei grundsätzlich an Folgendes erinnert: Nach einem für moderne Lyrik grundlegenden Literaturverständnis, das von den russischen Formalisten (Šklovskij u.a.) ausgegangen ist und das sich in vielen neueren Theorien aufgenommen findet, hat schöne Literatur vor allem die Funktion der Entautomatisierung von Wahrnehmung ${ }^{3}$. Diese lässt eine grundständige Verfremdung des verwendeten Wortmaterials entstehen und macht so Vertrautes wieder ,neu' beziehungsweise ,sichtbar', nachdem alltägliches Sprechen Wörter und Wendungen kaum noch eigentlich wahrnehmen und in einer gleichsam ,automatisierten" Verwendung verblassen lässt. Moderne und postmoderne Lyrik mit ihren zahlreichen Experimentalformen ist insofern herausragendes poetisches Medium, und Lyrik sodann, die sich dezidiert mit dem Fremden als Person, Sozialphänomen, Orts-, Sach- und zumal Kulturgegenstand auseinandersetzt, oder mit Fremd- und Eigenheit überhaupt, erscheint in diesem Zusammenhang geradezu als eine Potenzierung dieser Grundfunktion von der Literatur. Reiselyrik schließlich, verstanden als Literatur zur (in der Moderne stark subjektivierten, postmodern auch dekonstruktiven) Auseinandersetzung mit Fremdheit, wirkt in dieser Perspektive als entautomatisierend per se: in der basalen Sprachbewegung, aber auch der Bewegung durch Zeit, Raum und Soziokulturen, denn Reiselyrik setzt sich genuin dem Fremden aus und setzt es in Beziehung zum Subjekt der Wahrnehmung beziehungsweise zur Sprache der Auseinandersetzung und deren sozialem, kultu-

2. - Nicht eigens behandelt wird das Schaffen Durs Grünbeins, dessen zahlreiche Lyrik und (essayistische) Prosa mit synchronischen und diachronischen Bewegungen v.a. durch das geschichtsträchtige Italien beziehungsweise das Römische Reich und seine imperiale Kultur (vgl. beispielsweise D. Grünbein, Aroma. Ein römisches Zeichenbuch, Berlin, Suhrkamp, 2010, und zuletzt, in Teilen, ders., Zündkerzen. Gedichte, Berlin, Suhrkamp, 2017, oder für seine Prosa: ders., Antike Dispositionen. Aufsätze, Frankfurt a.M., Suhrkamp, 2005) zu dem bereits am meisten Beachteten im Bereich der Reiseliteratur im weitesten Sinne gehören; vgl. etwa Ruth J. Owen, „A Poetics of Presence. Travel Cycles in Aroma and Lob des Taifuns", in: Michael Eskin, Karen Leeder, Christopher Young (Hrsg.), Durs Grünbein. A Companion, Berlin, Boston, de Gruyter, 2013, S. 181-203.

3. - Vgl. etwa Viktor Šklovskij, „Die Kunst als Verfahren“, in: Jurij Striedter (Hrsg.), Russischer Formalismus. Texte zur allgemeinen Literaturtheorie und zur Theorie der Prosa, München, Fink, 1971, S. 3-35. 
rellem etc. Hintergrund. In diesem Sinne äußern sich auch Lyrikerinnen und Lyriker der Gegenwart, so etwa Paul-Henri Campbell in einem kurzen Statement mit den zugleich wörtlich und übertragen gemeinten Thesen: „Reisen ist für mich der Urstoff der Lyrik“, ,Dichter sind rastlose Ästheten“ und ,[ihre Werke] sind für mich eine bestimmte Art des Reisens; ein Unterwegssein ohne Grund, ohne Zweck, auch ohne Ziel"“4.

Die Entautomatisierung funktioniert freilich nur solange, wie es nicht zu einer Re-Automatisierung der sprachlichen und literarischen Mittel selbst kommt. Dafür sind ständige neue Oppositionsbildungen innerhalb der betreffenden literarischen Reihe vonnöten (nach Tynjanov u.a. $)^{5}$. Von daher müssen immer neue Ausdrucksformen in Absetzung von den vorangegangenen angestrebt werden, d.h. in einer modernetypischen Abweichungsästhetik. Will man Reiselyrik der letzten zwei Jahrzehnte im deutschsprachigen Raum insgesamt verorten, ist also nicht zuletzt $\mathrm{zu}$ fragen, welche Auseinandersetzung mit Fremdem/ Fremdheit in ihr zu finden ist und welche Absetzbewegungen innerhalb ihrer literarischen Reihe stattfinden. Um diese Fragen an den genannten Beispielautorinnen und -autoren untersuchen zu können, sei jedoch zuerst in der gebotenen Kürze skizziert: zum Ersten, wie es um den Forschungsstand zur deutschsprachigen Reiseliteratur im Allgemeinen und Lyrik-bezogen steht, und zum Zweiten, welche literarhistorischen Entwicklungen der deutschsprachigen Nachkriegszeit als relevante Etappen der literarischen Reihenbildung angesetzt werden könnten.

\section{Deutschsprachige Reiseliteratur und Lyrik seit 1945}

Grundlegende Arbeiten, die den literarischen Reisebericht zum Thema haben, stammen von Peter J. Brenner, der für diese Textsorte vornehmlich Vor- und Frühmoderne als Konjunkturphasen ansieht, weil mit dem Aufkommen des Massentourismus diese Gattung einen nachhaltigen Bedeutungsverlust erlitten habe ${ }^{6}$. Sodann hat sich eine breite Forschung zu zahlreichen Genres einer Reiseliteratur im weiteren Sinn etabliert ${ }^{7}$, und in jüngerer Zeit ist zudem das 20. Jahrhundert stärker in den Fokus gerückt, ja selbst das beginnende 21. Jahrhundert wurde

4. - Paul-Henri Campbell, „Beim Durchbrechen der Schallmauer“, Das Gedicht, 21/2013, S. 122 (Hervorhebung im Original).

5. - Vgl. etwa Jurij Tynjanov, „Über die literarische Evolution“, in: J. Striedter, Russischer Formalismus..., a.a.O., S. 433-461.

6. - Vgl. Peter J. Brenner (Hrsg.), Der Reisebericht. Die Entwicklung einer Gattung in der deutschen Literatur, Frankfurt a.M., Suhrkamp, 1989; ders., Der Reisebericht in der deutschen Literatur. Ein Forschungsbericht als Vorstudie zu einer Gattungsgeschichte, Tübingen, Niemeyer, 1990.

7. - Für einen Überblick vgl. Hans-Wolf Jäger, „Reiseliteratur“, in: Georg Braungart u.a. (Hrsg.), Reallexikon der deutschen Literaturwissenschaft, Bd. 3: P-Z, Berlin/New York, de Gruyter, 2010, S. 258-261. 
punktuell bereits untersucht ${ }^{8}$. Es wird dabei hervorgehoben, „dass die neuere Reiseliteratur durch eine Vielzahl von Mobilitätsmustern und Schreibverfahren geprägt ist, die sich gegenläufig zu [...] Brenners These vom Funktionsverlust des Reisens und der Reiseliteratur verhalten.“9

Was das Spektrum der zu erfassenden Genres bzw. Textsorten betrifft, wird gerne darauf hingewiesen, dass neben zahlreichen Prosaformen auch Lyrik begegne ${ }^{10}$, de facto werden jedoch lyrische Texte so gut wie nicht weitergehend beziehungsweise systematisierend analysiert ${ }^{11}$. Eine wichtige Ausnahme stellt eine Monographie von Jan Röhnert dar, die sich Rolf Dieter Brinkmanns antitraditionalistischer, eine autonome Fremdheitserfahrung suchende „Reiselyrik“ bzw. „Reisepoesie“ widmet. Diese ventiliert sogar das Fehlen eines stabilen Fachbegriffs für lyrische Dichtungen zu Reisethemen und plädiert schließlich gut nachvollziehbar im Wesentlichen für die Verwendung des Terminus „Reisegedicht“, je nach Kontext neben „Reiselyrik“ bzw. „Reisepoesie“, insbesondere im Gegensatz zu dem vagen (und unter Umständen sogar irreführenden) Begriff der „Ethnopoesie“12. An diese Begriffsentscheidung, aber auch an Röhnerts BrinkmannAnalysen wird im Folgenden u.a. angeschlossen. Mit Blick auf die literarische Reihe im näheren Vorfeld der hier interessierenden Werke sind zum einen die gängigen Annahmen von Phasen der deutschsprachigen Nachkriegsliteratur und ihrer Lyrik in Anschlag zu bringen ${ }^{13}$.

8. - Vgl. etwa Alfred Opitz, Reiseschreiber. Variationen einer literarischen Figur der Moderne vom 18.-20. Jahrhundert, Trier, WVT, 1997; Ulla Biernat, ,Ich bin nicht der erste Fremde hier". Zur deutschsprachigen Reiseliteratur nach 1945, Würzburg, Königshausen \& Neumann, 2004, oder jüngst und ausführlich: Michaela Holdenried, Alexander Honold, Stefan Hermes (Hrsg.), Reiseliteratur der Moderne und Postmoderne, Berlin, Schmidt, 2017.

9. - Michaela Holdenried, Alexander Honold, Stefan Hermes, „Reiseliteratur der Moderne und Postmoderne. Zur Einführung“, in: Dies. (Hrsg.), Reiseliteratur der Moderne ..., a.a.O., S. 9-16, hier S. 12.

10. - S. etwa die Aufzählung ebd., S. 11: ,[Reiseliteratur] umfasst demnach sowohl Reisehandbücher und wissenschaftliche Reisewerke, etwa Forschungstagebücher, als auch dezidiert literarische Reisebeschreibungen sowie Romane, Erzählungen, Essays und Gedichte [!], in denen tatsächliche oder erfundene Reiseerlebnisse gestaltet werden und für die eine Dominanz der ästhetischen Formung des Materials zu konstatieren ist"“.

11. - Vgl. etwa im negativen Sinne sogleich bei M. Holdenried, A. Honold, S. Hermes (Hrsg.), Reiseliteratur der Moderne ..., a.a.O. oder auch in der erwähnten, umfassenden Monographie von U. Biernat, ,Ich bin nicht der erste Fremde hier “..., a.a.O., und in H.-W. Jäger, „Reiseliteratur“, a.a.O., wird nicht ein einziges Mal explizit auf lyrische Reiseliteraturformen hingewiesen.

12. - Vgl. Jan Röhnert, Meine erstaunliche Fremdheit! Zur poetischen Topographie des Fremden am Beispiel von Rolf Dieter Brinkmanns Reiselyrik, München, Iudicium, 2003, und zu der genannten Begriffsfrage S. 38f., v.a. Fußnoten 23 und 26.

13. - Vgl. für übliche Phasierungen von 1945 bis in die 1990er Jahre etwa Hermann Korte, Deutschsprachige Lyrik seit 1945, 2., völlig neu bearb. Aufl., Stuttgart, Weimar, Metzler, 2004, oder Stefan Elit, Lyrik. Formen - Analysen - 
Zum anderen kann für die Reiseliteratur dieser Zeit(en) auf die Überblicksstudie von Ulla Biernat rekurriert werden, denn diese stellt die Grundannahmen bereits Reiseliteratur-bezogen schärfer heraus und wendet sich ihrerseits gegen Brenners (und anderer) Infragestellung der kulturellen Relevanz moderner Reiseliteratur.

Die erste Ergebnisperspektive Biernats bezieht sich dabei auf die gesellschaftlicheEntwicklung von Reisen und Tourismus; demnach habe sich schon mit den 1950er Jahren ,das Reisen als soziale Praxis ausdifferenziert: von der eskapistischen Italiensehnsucht über die Etablierung des Massentourismus in den sechziger Jahren, zum alternativen und sanften Tourismus der siebziger Jahre bis zu den Erlebniswelten, die seit den achtziger Jahren zeitliche und räumliche Fremderfahrungen simulieren." 14 Die Nachkriegszeit habe das Reisen entsprechend diskursiviert, d.h. ,auf den Kulturpessimismus und Eskapismus der fünfziger Jahre folgt die Verabschiedung des Exotismus und des authentischen Erlebens der Ferne“, während Biernat in den 1970er Jahren ,das Reisen im Dienst individualistischer Selbsterfahrungssuche und der wissenschaftlichen Fremdheitsforschung" sieht und in den 1980er Jahren ,als Zeichenrelation zwischen Authentizität und Simulation spielerisch dekonstruiert“. Die 1990er Jahre wiederum ,,[ließen] sich als ironischer Erlebnistourismus [...] vor dem Hintergrund der Globalisierung [charakterisieren]"15.

Weitergehend resümiert Biernat mit Bezug auf Konstruktionen und Relationierungen von Selbstund Fremdem im Laufe der Jahrzehnte, ohne in teleologischer Perspektive zu werten. Sie sieht in den Reisebereichten der 1950er Jahre ,das Andere als Projektionsfläche historischer Nostalgie, politischer Utopien oder literarischer Aneignungsstrategien", während in den 1960er Jahren Fremdes antithetisch zum Eigenen kodiert werde; dabei stünden auf der Seite des Fremden ,entweder die gesellschaftlichen Zustände im Heimatland des Autors oder die Folgen von Kolonialismus und Imperialismus im fremden Land selbst". In den 1970er Jahren erfahre diese Antithetik sodann eine Ausweitung auf das Grundverhältnis von Selbst und Anderem, wobei die Funktion des Anderen die eines Spiegels respektive einer Selbsterfahrungsgrenze sei. Wiederum forciert sieht Biernat diese Entwicklung in den 1980er Jahren durch eine Auflösung der Antithetik: „Das Andere wird entweder durch eine metafiktionale Textkonstruktion des Anderen ersetzt oder durch eine selbstreflexive Verstehensanstrengung [...] relativiert". In den neunziger Jahren schließlich finde sogar eine Auflösung der asymmetrischen Relationierung von Selbst und Anderem statt,

Gattungsgeschichte, München, Fink, 2008, S. 182-212.

14. - U. Biernat, ,Ich bin nicht der erste Fremde hier“, a.a.O., S. $209 f$.

15. - Ebd., S. 210. 
weil die Narration als solche nicht mehr gattungstypisch erfolge. Übrig bleibe das Andere als „Zeichenraum"16. Es handelt sich hier um Thesen v.a. für Reiseliteratur in Prosa, lyrische Texte werden jedoch punktuell mitbehandelt beziehungsweise plausibel als analog sich entwickelnde Gattungsformen angenommen. Diese Thesen sollen daher bei den folgenden Sondagen in die Reiselyrik der Gegenwart für eine erste literarische Reihenbildung als potenzielles Vorfeld angesetzt werden.

\section{Uwe Kolbes, Nachholen'von Reiseerfahrung und sein mythisches Spiel damit}

Uwe Kolbes lyrisches Werk zerfällt bis dato in mindestens zwei Hälften, und zwar in eine Werkgruppe bis kurz nach dem Ende der DDR, die auf den Spuren von Expressionismus (Heym, Trakl) oder Hermetismus (Huchel u.a.) wandelte, d.h. von Kolbes Debütband Hineingeboren (1980) bis hin zur älteren Werkschicht in Vaterlandkanal. Ein Fahrtenbuch (1990), und in eine stetig wachsende Gruppe von Gedichtbänden ,seitdem', die zahlreiche neue lyrische ,Tonarten' aufweisen, d.h. von Vaterlandkanal in seinen neueren Teilen bis hin zu Kolbes jüngstem Band Psalmen (2017) ${ }^{17}$. Letztere Gruppe weist mittlerweile verschiedene thematische Schwerpunkte auf, etwa eine sich ab den frühen 2000er Jahren intensivierende und aktuell wieder etwas abklingende Auseinandersetzung mit der griechisch-römischen Antike, und könnte danach ausdifferenziert werden. Dieses interessiert hier jedoch weniger als die sie verbindende Linie reiselyrischer Themen, die im Folgenden auswahlweise betrachtet werden soll18. Den Auftakt bildet der Band Vaterlandkanal mit denjenigen Gedichten, die Kolbe offensichtlich verfasst hat, seitdem er die DDR ab Mitte der 1980er Jahre mit Reisevisa länger verlassen durfte (und 1987 auch dauerhaft als Wohnsitz aufgegeben hat) ${ }^{19}$. Der prägnante Untertitel des Bandes,

16. - Alle Zitate ebd., S. 214f.

17. - Zu Autor und Werkentwicklung bis Anfang der 2010er Jahre vgl. Verf. (Hrsg.), ,... notwendig und schön zu wissen, auf welchem Boden man geht". Arbeitsbuch Uwe Kolbe, Frankfurt a. M., Lang, 2012 bzw. im Kurzüberblick Verf., „Uwe Kolbe: Gedichte", in: Marion Bönninghausen, Jochen Vogt (Hrsg.), Literatur für die Schule. Ein Werklexikon zum Deutschunterricht, unter Mitarb. v. Dirk Hallenberger, Paderborn, Fink, 2014, S. 498-501.

18. - Als eine erste Sondage zum Thema, die im Folgenden aufgegriffen und weitergeführt wird, vgl. Verf., ,Von der DDR in Gegenwart und Antike. Zur lyrischen Weltenreise des Uwe Kolbe“, in: Hermann Korte, Henrieke Stahl (Hrsg.), Gedichte schreiben in Zeiten der Umbrüche: Deutsche und russische Lyrik seit 1989, München, Lang, 2016, S. 247-258.

19. - Für biographische Angaben vgl. hier und im Folgenden den von Uwe Kolbe selbst verfassten Eintrag zur eigenen Person: „Uwe Kolbe“, Literaturport.de (http:// www.literaturport.de/Uwe.Kolbe, Zugriff am 17.10.2018). 
Ein Fahrtenbuch, weist auf das Aus-Reisen eines DDR-Bürgers schon generell hin, und als konkrete Ziele finden sich neben Hamburg, dem neuen Wohnort Kolbes, als faszinierender Erfahrungsort fremder Kultur Amsterdam ${ }^{20}$. Weitere Stufen der Fremdheitserfahrung boten sich Kolbe durch Stipendien in Austin/Texas (1989) sowie in der Villa Massimo Rom (1992), und der Romaufenthalt zeitigte auch zahlreiche literarische Reflexe in den Gedichtbänden Nicht wirklich platonisch (1994) und Vineta (1998). In letzterem Band wird sogar bereits ein Zwischenfazit für die neuen Reise-Erfahrungen gezogen, und dabei auch auf den Punkt gebracht, dass hier ein Dichter-Ich unterwegs ist, das von der spezifischen DDR-Erfahrung lange Zeit vorenthaltener, Reisen ins kapitalistische Ausland' und einem damit verbundenen ,Erlebnisstau' geprägt ist und das sich daran bis auf Weiteres abarbeiten wird. Dieses offene Fazit findet sich ausführlich im Gedicht mit dem prägnanten Titel „Was hab ich noch nachzuholen“, das daher komplett zitiert sei:

Fast jeden Ort hatte ich nachzuholen, fast jeden Anblick. So einen gewissen blauen Berg hatte ich nachzuholen.

Wo hernach? Doch eigentlich hervor.

Ein Venedig und ein Comersee und ein Lugano und ein che bello im Original.

Amsterdam habe ich zeitig nachgeholt, Kopenhagen brachte ich ähnlich hinter mich und meine Frau, die noch mehr nachzuholen hatte.

Was habe ich noch nachzuholen?

So viel hatte ich gelesen über Eastside und Westside New York hatte ich unbedingt nachzuholen, nach Chicago, nach San Francisco, nach dem Grand Canyon undsoweiter. Oder wars andersherum?

Erst holte ich den Ku'damm nach dem Savignyplatz. Erst holte ich das Oberhalb der Mauer nach, in der SBahn, nach dem jahrzehntelangen Vor oder Hinter.

Was hab ich noch nachzuholen: Paris und Provence und Rom sind bereits nachgeholt, hab ich abgeholt.

Wem hab ich was nachzuholen?

Wie hol ich die liebe Gewißheit aber?

Wie hol ich das Kind nach, das hätt mir so wohlgetan?

Aber das ist ein Durchgangsstadium ${ }^{21}$.

20. — Die Stadt wurde von Kolbe bereits seit 1986 auf Einladungen für ihn als Autor hin besucht (vgl. ebd.); für Gedichte zu Amsterdam bzw. den Niederlanden vgl. etwa „Inkarnationen“ und „Ein fahrender Deutscher in Holland“, in: Uwe Kolbe, Vaterlandkanal. Ein Fahrtenbuch, Frankfurt a. M., Suhrkamp, 1990, S. 24 u. 25.

21. - Uwe Kolbe, Vineta, Frankfurt a. M., Suhrkamp, 1998, S. 18 (Hervorhebung im Original). 
Schaut man genauer auf Kolbes Reiselyrik dieser Zeit, zeigt sich jedoch nicht nur die Perspektive des ,entlassenen ${ }^{6}$ DDR-Bürgers, sondern Kolbes Werke nehmen auch spezifische Linien der oberhalb skizzierten Reiseliteratur der Nachkriegszeit auf. Am deutlichsten wird dies mit Bezug auf die Stadt Rom, die etwa in Nicht wirklich platonisch, zweites Kapitel ${ }^{22}$, in einer hybriden und morbiden Präsenz von (zumal antiker) Geschichte und Gegenwart entgegentritt, wie sie zuvor insbesondere Rolf Dieter Brinkmann Anfang der 1970er Jahre in Absetzung von einem Italienkult seit Goethe und bis in die 1960er Jahre literarisiert hatte. $\mathrm{Zu}$ verweisen ist in diesem Zusammenhang insbesondere auf Kolbes Gedicht „Arkadien“23. Dort wird das in der Gegenwart sehr heruntergekommene und von Mafiaproblemen geprägte Ostia als ein Anti-Arkadien im Sinne von Brinkmanns Westwärts 1 \& 2 (1975) ,besungen' und Brinkmanns hoch skeptische Perspektive auf Rom und seine Umgebung als exemplarischer Ort der ,westlichen' Zivilisation erneuert und sozusagen in die 1990er Jahre fortgeführt. Indirekter in stilistischer wie reisethematischer Brinkmann-Nachfolge steht sodann das Prosagedicht „Zueignung in Rom“ in demselben Bandkapitel24, das mit seinem schnoddrig-pathetischen und kaskadenartigen Stil der Stadtbeschreibung sowie in einigen thematischen Details nicht nur an Westwärts $1 \& 2$, sondern auch an Brinkmanns Prosaband Rom, Blicke (1979) erinnert ${ }^{25}$ und sich produktiv in dessen Perspektiven einschreibt.

Wenn sich Kolbe in den 2000er Jahren literarisch der griechischrömischen Kultur und ihrer Mythologie zuwendet und auch weitere Gegenden (Europas) thematisiert, ergeben sich Blicke auf von Geschichte ,durchschossene' fremde Gegenwart, die über einen skeptischen Blickwinkel Brinkmanns hinausgehen. Insbesondere der Gedichtband Heimliche Feste (2008) ${ }^{26}$ präsentiert eine große reisethematische Breite und ist dabei immer wieder antik-mythologisch aufgeladen; fast alle Bandkapitel erscheinen auf Bewegung in Räumen und Zeiten bezogen: So zeichnet Kapitel „I Sailor's Home“ $(H F, 7-20)$ den Lyriker gleichsam als singenden Seemann; ,II In Pfalzen“" zeigt sich mythisierend vor dem Hintergrund von Kolbes Aufenthalten im Künstlerhaus Edenkoben bei Landau in der Pfalz (HF, 21-29); „III In Thrakien“ $(H F, 31-44)$ gründet

22. - Vgl. Uwe Kolbe, Nicht wirklich platonisch, Frankfurt a. M., Suhrkamp, 1994, S. 23-38.

23. - Vgl. ebd., S. 35; das Gedicht endet sogar explizit mit der ironisch-pathetischen Anrufung ,und o Rolf-Dieter, bewahre uns unser Arkadien / fuori le mura, hier wie nirgendwo sonst in den Himmeln.“.

24. - Vgl. ebd., S. 25.

25. - Vgl. hierzu bereits Verf., „Von der DDR in Gegenwart und Antike“, a.a.O., S. $253 f$.

26. - Uwe Kolbe, Heimliche Feste, Frankfurt a. M., Suhrkamp, 2008 (weitere Verweise auf diesen Band mit Sigle $H F$ und Seitenangaben im Text). 
auf einem Stipendienaufenthalt in Sofia und im Süden Bulgariens, der sich als Ort und Kulturerbe des antiken Thrakien versteht; „IV An Orten" $(H F, 45-60)$ bezieht sich vor allem auf Städte und Landschaften der ehemaligen DDR, zu denen Kolbe besondere Bezüge hat; „V In Nächten" $(H F, 61-71)$ weist verschiedene, auch nochmals Bulgarienbezogene Reisethemen auf, und „VI Im Norden“ $(H F, 73-83)$ schließlich versammelt Gedichte zu Norddeutschland(aufenthalten des Autors).

Herausgegriffen sei aus dieser Kapitelreihe „III In Thrakien“, weil zumal in diesem Abschnitt eine neue, postmodern-spielerische Inbeziehungsetzung von antik-mythischer Vorzeit (insbesondere in der Gestalt des thrakischen Sängers Orpheus), orthodox-christlicher Tradition und der Gegenwart Bulgariens als eines - wie Kolbes DDR-Heimat - postsozialistischen Landes stattfindet, die über die Skepsis Brinkmanns und dessen implizite Ablehnung, schöner' antikmythischer Deutungen hinaus heiter-ironisch gestimmt ist ${ }^{27}$. Gezeigt werden kann dies exemplarisch an dem Gedicht „Nach einer Inschrift“, das Kolbes Reisegefährten in Bulgarien, dem Berliner Schriftsteller und Literaturhausleiter Ernest Wichner, gewidmet ist ${ }^{28}$. Auf Reisen ist in diesem Gedicht ein lyrisches Wir, das wohl zuerst als Kolbe und Wichner, aber auch als das Kollektivum reisender Menschen unserer Gegenwart zu deuten ist. Dessen Reiseperspektive ist auf den ersten Blick von einer großen Frustration geprägt, weil - wie Reiseliteratur seit den 1960er Jahren auf unterschiedlichste Weise reflektiert - eine originäre eigene oder sogar originell aufzuzeichnende Erfahrung nicht mehr möglich erscheint; das Gedicht beginnt mit den Versen: „Vor uns schon wieder - das ist ungeschickt. / Nach uns noch immer - das ist ärgerlich.“ Vor allem das „Nach uns“ ist in zahlreichen Folgezeilen des Gedichts anaphorisch an den Anfang gesetzt und verkettet eine große Reflexion der eigenen Belanglosigkeit. Diese ergibt sich mit Blick auf die endlose Zahl weiterer, vielleicht sogar erlebnisreicher Reisender, aber auch in dem Bewusstsein, dass jegliches eigenes Tun ein bloßes Durchreisen ,im Wirtshaus unserer Gegenwart“ $(H F, 34)$ sei.

Dem scheint eine Art gelassener Dauer der besuchten Kultur- und Naturorte sowie eine Verbindung der Menschen Bulgariens mit ihrer

27. - Zu Heimliche Feste generell und Kolbes Thrakien-Arbeiten der 2000er Jahre in Prosa und Lyrik vgl. im Überblick Verf., „Orpheus singt: von Ölbäumen und anderem. Zur Antikerezeption im Werk Uwe Kolbes“, in: Verf., Kai Bremer, Friederike Reents (Hrsg.): Antike - Lyrik - Heute. Griechisch-römische Antike in deutschsprachiger Lyrik und Altphilologie der Gegenwart, Remscheid, Gardez! 2010, S. 261-283, hier S. 273-281.

28. - Das Gedicht vgl. in $H F, 33$ f., für die gemeinsame Reise von Kolbe und Wichner vgl. Ernest Wichner, „Brief an Uwe“, in: Verf. (Hrsg.), ,... notwendig und schön zu wissen, auf welchem Boden man geht", a.a.O., S. 245-248 (mit einem Foto der beiden vor Ort). 
,großen' griechischen Urzeit gegenüber zu stehen: Selbst ein einfacher, korpulenter Automechaniker ist noch ,,in der Dicke kundig / des Wegs zum thrakischen Heiligtum“, und „Nach uns sind Spindel, Spule, Spülicht / am Straßenrande ganz die alten, / die Moiren unbewegt" $(H F, 33)$. Doch die reiseliterarische Vergleichsfigur des auf glückliche Weise zeitlos-ewigen Anderen in einfacheren Lebensverhältnissen wird nicht nur melancholisch, sondern auch schalkhaft aufgerufen. Darauf weist schon die saloppe Bezeichnung des Automechanikers als „ein Dicker" hin, aber auch die abrupte Umkehrung von ,ganz die alten" in den Folgezeilen, wenn es scheinbar pathetisch über weitere mögliche ,schön-antikische“ Reise-Erfahrungen heißt: ,nach uns wird wirklich alles anders sein, / der Wein, das Rhy ton anders vollgeschenkt" $(H F, 33)$, und kurz darauf wird selbst diese ,Hoffnung' wieder umgestoßen: „nach uns, ach was -, / nach uns derselbe Bilderklau“ $(H F, 34)$. Die in unserer Gegenwart und Zukunft, von unserer Zivilisation nicht mehr zu erreichende originale Erfahrung erscheint zunächst ähnlich frustrierend wie in der reiseliterarischen Reihe seit spätestens den 1970er Jahren, und auch das Andere fremder Kulturen wird nur noch alludierend gegenübergestellt. Kolbe-Gedichten wie diesem ist jedoch eigen, dass die ernüchternden Einsichten, die implizit die Frage nach der Entautomatisierung von Wahrnehmung überhaupt stellen, mit einem heiter-ironischen Habitus umspielt werden und so vielleicht auch ein letztes Interesse an Literatur mit Reisethemen erzeugen. Ein Gedicht mit dem ,ehrwürdigen“ Titel „Nach einer Inschrift“" wird so zu einer zugleich ernsten und heiteren Daseinsreflexion des DichterIchs und für , uns', die die Aporien postmoderner Reiseliteratur sozusagen locker transzendiert ${ }^{29}$.

\section{Jan Wagners behutsame, Probebohrungen“ ins Fremde als Faszinosum und Skurrilität}

Jan Wagner, 1971 in Hamburg geboren, hat ebendort sowie in Dublin und Berlin Anglistik studiert, und ist auch als Übersetzer vor allem von zeitgenössischer englischer, irischer und amerikanischer Lyrik tätig; er widmet sich also Fremdem auf diese Weise

29. - Nochmals (buchstäblich) weitere Reiseziele finden sich in neueren Gedichtbänden Kolbes, d.h. jeweils in einem Kapitel in den Lietzenliedern (vgl. die Nordamerika-Gedichte in Kapitel II von ders., Lietzenlieder, Frankfurt a. M., Fischer, 2012, S. 17-26), und in den Gegenreden (vgl. den Abschnitt „Transit“, zu Australien und Neuseeland, in Kapitel IV von ders., Gegenreden, Frankfurt a. M., Fischer, 2015, S. 87-95). Diese Thematisierungen bzw. Literarisierungen müssen allerdings weiteren Forschungen vorbehalten bleiben, um an dieser Stelle die Auseinandersetzung mit einem einzelnen Autor nicht zu überdehnen. 
bereits grundständig literarisch ${ }^{30}$. In dichter Folge hat Wagner seit seinem 2001er Debütband Probebohrung im Himmel Gedichtbände im (Bloomsbury-)Berlin-Verlag bzw. bei Hanser vorgelegt, so Guerickes Sperling (2004), Achtzehn Pasteten (2007), Australien (2010), Die Eulenhasser in den Hallenhäusern. Drei Verborgene (2012) und zuletzt Regentonnenvariationen (2014) sowie die Auswahlsammlung Selbstporträt mit Bienenschwarm (2016). 2011 hat er außerdem unter dem Titel Die Sandale des Propheten einen Band mit Vorträgen und Essays veröffentlicht, der laut Untertitel Beiläufige Prosa versammelt ${ }^{31}$. Diese Prosa ist dabei „beiläufig“ insofern, als sie sozusagen ,neben' Wagners Lyrik ,herläuft' und sie sowie seine Perspektive auf Poesie überhaupt erläutert. Eine gewissermaßen ,erste ${ }^{6}$ Definition von Lyrik findet sich im Titelbeitrag des Essaybands: „Das Gedicht ist auf herrliche Art und Weise vollkommen nutzlos - so nutzlos wie ein Lachen nutzlos ist, ein jähes Glücks- und Hochgefühl, ein absichtsloses Spiel“32. ,Lachen" und ,Hochgefühl' als zwei Facetten hat denn auch bereits Wagners Debütband gesetzt, wenn er Probebohrung im Himmel verspricht: ,Himmel‘ stünde insofern für einen ,hochgestimmten“ bzw. ,hohen Ton' und damit klassischerweise für eine Auseinandersetzung mit ,erhabenen' Themen; die ,Probebohrung' weist darauf hin, dass nur vorsichtige und präzise Sondagen vorgenommen werden. Die etwas gewagte Wortverbindung als Ganze wirkt dem gegenüber ironisierend und kündigt einen (auch) humoristischen Umgang mit ernsten Themen an. Diese Junktur, die mutatis mutandis Uwe Kolbes doppeltem Ton nicht unverwandt erscheint (aber davon noch zu trennen sein wird), ließe sich an zahlreichen Texten dieses und weiterer Lyrikbände Wagners zeigen ${ }^{33}$. Soll es jedoch zugleich um die Frage von Reiselyrik gehen, kommt unweigerlich der Band Australien in den Blick, der in vier Kapitel nach den Himmelsrichtungen gegliedert ist, gefolgt von einem Kapitel unter dem Bandtitel ${ }^{34}$. ,Ausgerichtet ${ }^{\star}$ sind die fünf

30. - Für Informationen zur Person und zur Werkentwicklung vgl. generell Frieder von Ammon (Hrsg.), Jan Wagner, Text + Kritik, 210/2016; Pia Elisabeth Leuschner, ,Jan Wagner - Essay“, in: Hermann Korte (Hrsg.), Kritisches Lexikon zur deutschsprachigen Gegenwartsliteratur (www.munzinger.de, Zugriff am 30.10.2018) sowie Jan Wagner, „Jan Wagner“, Literaturport.de (http://www.literaturport.de/Jan. Wagner, Zugriff am 30.10.2018).

31. - Jan Wagner, Die Sandale des Propheten. Beiläufige Prosa, Berlin, Berlin/ Bloomsbury 2011; 2017 folgte mit dem gleichen Untertitel sodann die noch größere Vortrags- und Essay-Sammlung Der verschlossene Raum am selben Ort.

32. - J. Wagner, Sandale des Propheten, a.a.O., S. 20.

33. - Exemplarisch sei verwiesen auf ein Gedicht in: Jan Wagner, Probebohrung im Himmel, Berlin, Berlin/Bloomsbury 2001, S. 22: „Im Sommer '99“, in dem ,hoher Ton' und feiner Humor in einem virtuosen Wechselspiel zu finden sind.

34. - Vgl. Jan Wagner, Australien, Berlin, Berlin/Bloomsbury 2010 (weitere Verweise auf diesen Band mit Sigle $A$ und Seitenangaben im Text). 
Kapitel dabei nach dem persönlichen Kompass des Autorinteresses: Im „Süden“ liegt vor allem das Mittelmeer ( $A, 7-22)$, im „Westen“ zuerst das südliche und dann das nördliche Amerika ( $A, 25-41)$, im „Osten“ das östliche Europa $(A, 43-57)$ und im „Norden“ Jan Wagners norddeutsche Kindheitsheimat sowie Skandinavien $(A, 69-75)$. Dem titelgebenden Kontinent ist das letzte Kapitel $(A, 77-99)$ jedoch nur auf den ersten Blick gewidmet, denn schon ein dem ganzen Band vorangestelltes Zitat von Álvaro de Campos (einem Pseudonym von Fernando Pessoa) legt eine andere Fährte: „Man ist glücklich in Australien, / sofern man nicht dorthin fährt.“ $(A, 5)$ Dieses wagnerisch humorvolle und ernste Bonmot klingt fast nach einer Absage an das Reisen (und damit auch an die Reiselyrik) schlechthin, allerdings sind die vier folgenden Kapitel zumindest zahlreichen anderen Reisezielen gewidmet. Das Kapitel "Australien“" schließlich steht außerdem für eine Welt des ,Anderen“ schlechthin, versammelt es doch Gedichte, die diversem ,Fremdem gewidmet sind, bis hin zu einem Titelgedicht, das den ganzen Band beschließt und zeigt, wie schon die unmittelbare Umgebung für Kinder (und damit wohl auch poetologisch: für entsprechend, weltoffene' Lyrik) ein faszinierend fremder Kontinent sein kann und damit sozusagen ein Ersatz-Australien, in dem man ,glücklich` sein kann $(A, 98)^{35}$.

Tatsächliche Sondagen in einen anderen Kontinent, also jenseits des vertrauteren Europa, unternimmt insbesondere das zweite Bandkapitel, das die beiden Amerikas von der europäischen Warte aus als „Westen“ verortet, das sich seinen einzelnen Themen (Orten, Tieren, Menschen) jedoch behutsam und präzise in einer je eigens ,angemessenen Atmosphäre und lyrisch ganz unterschiedlich nähert, so etwa in dem Sonett ,tukan“ $(A, 27)$, in der Ode ,die missionsstation“ ( $A, 28 \mathrm{f}$.), in der „elegie für knievel“" $(A, 33)$, in dem volksliedartig strophischen ,[T]rapper"-Gedicht $(A, 34)$ oder in den mit Terzinen spielenden Gedichten ,ohio" und „murmeltier“ $(A, 35,36)$. Auffällig ist bei allen diesen Texten, dass sie als Momentaufnahmen respektive analytische Beschreibungen eines erreisten ,Fremden' erscheinen, wer hier jedoch reist und beschreibt, ist kaum oder gar nicht fokussiert, sprich: allenfalls ganz am Rand oder am ehesten am Gedichtende, so etwa in ,trapper“ oder „,die missionsstation“, begegnet ein lyrisches Ich, ein Du oder ein quasi reisegemeinschaftliches Wir, z.B. in „tukan“. Dieser Grundzug setzt Jan Wagners Reiselyrik bei allem ähnlichen heiter-ernsten Spiel von derjenigen eines Uwe Kolbe deutlich $\mathrm{ab}$, dessen prononcierte (Post-DDR-)Subjektivität für seine Reisegedichte meistens integral erscheint. Als etwas genauer zu betrachtendes Textbeispiel sei das prosalyrische, locker dreistrophige Gedicht

35. - Vgl. Gerhard Kaiser, „Children at work - Ein Versuch über Jan Wagners Programmgedicht ,australien“", in: Christoph Jürgensen, Sonja Klimek (Hrsg.), Gedichte von Jan Wagner. Interpretationen, Münster, Mentis 2017, S. 159-173. 
„vom lake michigan“ $(A, 37)$ herangezogen, in dem ein erhabenes Thema mit humorvollen Perspektivierungen beschrieben wird, und das metrisch wie in den metaphorisch gedeuteten Inhalten behutsam-tentativ verfährt, und zwar sozusagen wie die staunende Annäherung eines Besuchers an eine fremde Natur- und Kulturlandschaft. In dieser erlebt das Gedicht bzw. die Besuchsgemeinschaft zunächst eine Naturerhabenheit still, denn, so die erste Strophe:

die ganze nacht lang tobte der sturm ums weiße holzhaus, zusammengehalten von nichts als dem dünnen lampenschein seiner zimmer. die herbstlichen kronen der bäume am nächsten morgen wie eingeworfene kirchenfenster.

Mit Strophe zwei wendet sich der Blick einem Stück US-typischer Massenkultur an diesem See zu, das jedoch in der stürmischen Jahreszeit auf skurrile Weise bildhaft verfremdet erscheint:

der stillgelegte vergnügungspark

mit den seeschlangen seiner achterbahnen:

in guten sommern nähren sie sich

von all den gezuckerten schreien, nun stehen sie, starr vor dem winter.

Die dritte Strophe beschließt das Gedicht, vielleicht wie ein Reisebericht an Vertraute in der Heimat adressiert, mit einer weiteren stillen Naturbeobachtung, die Fremdes staunend in Bildern zu fassen versucht und dabei die Begrenztheit der eigenen Approximation mit ausstellt:

jeden abend, freunde, schneidet

die dämmerung die umrisse

von rehen aus den wäldern.

die langen gänge am strand entlang:

schwer zu sagen in diesem einsamen licht,

ob es ein schwarzbär ist, der ans ufer klettert,

oder ein stückchen treibholz,

zu einem schwarzbären verbrannt.

Die reiselyrische Auseinandersetzung erfolgt also wie bei Kolbe in einem heiter-ernsten Spiel, das die großen Phasen bzw. Linien der Nachkriegsliteratur schlichtweg hinter sich lässt. Im Gegensatz zu Kolbe scheint die eingeschriebene Subjektivität jedoch die Festlegung auf ein konturierteres lyrisches oder sogar Dichter-Ich zu vermeiden und eher ein allgemeines Staunen vor der Welt zu vertreten, das sich im besten Sinne als Grundlage der Entautomatisierung von Wahrnehmung durch Literatur verstehen lässt. 


\section{Ulrike Almut Sandigs infinite Suchbewegungen aus dem ,Dickicht}

DieAutorin wurde 1979 in Nauwalde in derNähe von Dresden geboren und ist aufgewachsen als Pastorentochter, was im DDR-Kontext bereits eine basale Fremdheitserfahrung implizierte, d.h. aus der Familie heraus eine weltanschauliche Andersheit gegenüber dem Staatssozialismus. Anfang der 2000er Jahre absolvierte Sandig zunächst in Leipzig ein Magisterstudium in Religionswissenschaft und Moderner Indologie, es folgte 2010 das Diplom des Deutschen Literaturinstituts ebendort. Erste literarische Werke machte sie 2001 auf Plakaten in der Stadt publik, und mit der Autorin und Musikerin Marlen Pelny präsentierte sie Texte auf Lesebühnen ${ }^{36}$. An Buchveröffentlichungen erschienen bislang die Gedichtbände Zunder (2005, überarbeitet 2009), Streumen (2007), und 2011 kamen in ihrem neuen Hausverlag Schöffling \& Co. der Gedichtband Dickicht und das CD-Album (zusammen mit Maren Pelny) Märzwald. Dichtung für die Freunde der Popmusik, heraus, das Vertonungen der drei genannten Gedichtbände enthält ${ }^{37}$. Der jüngste Gedichtband, ich bin ein Feld voller Raps verstecke die Rehe und leuchte wie dreizehn Ölgemälde übereinandergelegt (2016), war sogleich mit einer Auswahl von Vertonungen auf CD erhältlich. Ebenfalls bei Schöffling hat Sandig außerdem abwechslungsreiche Erzählbände vorgelegt, Flamingos (2010) und Buch gegen das Verschwinden (2015).

Sandigs lyrisches Schaffen in den ersten beiden Gedichtbänden ist von bis ins Hermetische gehenden Sprachexperimenten geprägt, dabei erscheint als Schlüssel zum Verständnis jedoch ein genaues Beachten des Sprachklangs. Zumal die Rezitation der Autorin selbst erschließt den Sinn z.B. der intensiven Sprachspiele um Phantasie und Erinnerung ${ }^{38}$. Die letzten beiden Gedichtbände (ganz zu schweigen von ihrer luziden Prosa) sind hingegen in Syntax, Semantik und Beat-artig melodischer Sprachrhythmik beeindruckend ,zugänglich` (aber keinesfalls simpli-

36. - Für Informationen zur Person und zur Werkentwicklung vgl. generell Mark Behrens, „Ulrike Almut Sandig - Essay“, in: Hermann Korte (Hrsg.), Kritisches Lexikon zur deutschsprachigen Gegenwartsliteratur (www.munzinger.de, Zugriff am 30.10.2018); Ulrike Almut Sandig, ,Ulrike Almut Sandig“, Literaturport.de (http://www. literaturport.de/Ulrike-Almut.Sandig, Zugriff am 30.10.2018) sowie die von der Autorin betriebene informative Website mit dem Titel Ulrike Almut Sandig. Autorin (www. ulrike-almut-sandig.de, Zugriff am 2.11.2018).

37. - Genuine Hörbücher bzw. Hörspiele hat Sandig bereits zuvor veröffentlicht, und zwar der tag, an dem alma pastillen kaufte (2006), Hushlittle Baby (2008) und Unter Wasser (2010). Auszüge aus fast allen Audio-Veröffentlichungen der Autorin finden sich in Ulrike Almut Sandig, „Audiothek“, in: Dies., Ulrike Almut Sandig. Autorin (www.ulrike-almut-sandig.de/audiothek, Zugriff am 2.11.2018).

38. - Vgl. M. Behrens, „Ulrike Almut Sandig - Essay“, a.a.O. 
zistisch) geworden. Der frühere der beiden Bände, Dickicht ${ }^{39}$, ist sodann auch derjenige, der für die Frage von Reisethemen in Sandigs Lyrik am interessantesten erscheint. Beim ersten Blick auf die Kapiteltitel fächert sich der ganze Gedichtband scheinbar geographisch auf wie bei Kolbe oder Wagner: Nach einem Prosamotto und einem Prologgedicht heißt der erste große Bandabschnitt „Norden“ (D, 9-37), der folgende Kurzabschnitt „Mitte der Welt" $(D, 39-41)$, ein wieder umfangreicher Abschnitt „Süden“ (D, 43-71), und beschlossen wird der Band von einem weiteren Kurzabschnitt, mit dem mathematischen Zeichen für Unendlichkeit als Titel: „, “( $D, 73-75)$. Bei genauerer Kontextbewertung schon des Prosamottos, Kafkas bekannter Kurztext „Wunsch, Indianer zu werden“ $(D, 5)$, gewinnt man jedoch den Eindruck, dass es anstelle von Reisen mit bestimmten Zielen (die in den Bandabschnitten grob geographisch gebündelt würden) um etwas Allgemeineres gehen könnte, nämlich eine forcierte und befreiende Bewegung ,ins Offene', aus dem ,Dickicht' des Bandtitels heraus. Dieser Impetus bestätigt sich peu à peu: Das Prologgedicht ,das hast du dir selbst gedichtet“ $(D, 7)$ beschreibt sozusagen eine globale Standortbestimmung des lyrischen Ichs als Dichterinnen-Ich, das am Ende vor allem feststellt: ,in dir / die Nadel, die zittert und immer hinzeigt / auf Norden, obwohl du nicht weißt, was da liegt.“ $(D, 7)$ Es gibt also metaphorisch gedeutet vor allem einen Orientierungswunsch im Sinne eines inneren Kompasses, der vielleicht für den Wunsch nach Welt- und Selbsterfahrung in einer generellen Bewegung, ins Nördliche‘ spricht.

Im sich anschließenden Bandabschnitt „Norden“ sind entsprechend nicht Reiseziele als Erfüllungsorte gesammelt, sondern persönliche und historische Erinnerungsstücke an Personen, Tiere, Dinge, Orte und Geschehnisse sowie Welt- und Selbstreflexionen, die als Orientierungsversuche lesbar sind und die allenfalls ganz grob in Deutschland bzw. dem nördlichen Mitteleuropa als Raum angesiedelt erscheinen. Unterstützt wird die generelle Bewegungsmetaphorik dabei von Gedichten wie „vom Himmel“ $(D, 27)$ und „,vom Flieger aus siehst du“ $(D, 28)$, die Flüge als solche ohne erkennbare Einzelziele thematisieren. Das letzte Gedicht dieses Abschnitts bereitet dann schon titelmäßig den Übergang zum nächsten Abschnitt vor: ,zur Mitte der Welt", und beschreibt, worum es bei dieser Bewegungsrichtung geht, nämlich ,den richtigen Weg zum gemeinsamen / Treffpunkt“ $(D, 37)$ $\mathrm{zu}$ finden. Metaphorisch gedeutet ergibt sich damit, dass die Bewegung des Ichs hier nicht nur weg-von geht, sondern auch an einen imaginären Ort des Zusammenkommens mit einem vermutlich geliebten Du, und dies bestätigt sich auch durch das Folgegedicht, das den einzigen Inhalt

39. - Vgl. Ulrike Almut Sandig, Dickicht, Frankfurt a. M., Schöffling \& Co, 2011 (weitere Verweise auf diesen Band mit Sigle $D$ und Seitenangaben im Text). 
des Bandabschnitts „Mitte der Welt“ bildet, sein Titel lautet: „in der Mitte des jeweils eigenen Körpers" $(D, 41)$. Imaginiert wird hier, dass sich in der Körpermitte von Ich und Du ein „Riss“ (im Sinne von Platons ,Kugelmensch') befinde, den es durch ein Wiedervereinigen, „wir nähen uns aneinander“ $(D, 41) \mathrm{zu}$, heilen“ gelte.

Wenn es von hier aus, sozusagen durch Physis und Psyche von Ich und Du hindurch, weitergeht in den „Süden“, finden sich grob gesprochen ähnliche Themen und Motive wie im „Norden“ wieder, die Frage nach ,Süden' als neuer Bewegungsrichtung wird aber durchaus auch angesprochen, so etwa durch das einzige (!) konkrete Reisegedicht, auf die Stadt „Sydney“ $(D, 45)$, und das Gedicht mit dem Titel „Süden“" verbindet explizit den Impetus des ,Wegwollens" mit der Frage, ob ein „Süden“, sozusagen in deutscher Tradition verheißungsvoll, als Sehnsuchtsort Erfüllung bringen kann; dem scheint jedoch nicht so zu sein, ,denn Süden steht / immer im Süden“ $(D, 66)$, sprich: ist nicht wirklich zu erreichen. In dieser Bildlinie lässt sich auch der letzte Bandabschnitt, „,“, als infinite Suchbewegung verstehen, dessen einziges Gedicht, „von allen guten Geistern“ $(D, 75)$, zwar vor allem das Bandende metaphorisiert (erste Gedichtzeile: „wenn das letzte Lied aus ist"), aber auch ein gleichsam unendliches Verklingen - dem Bandthema ,Sehnsuchtsbewegung aus dem Dickicht' gesellt sich so noch einmal die für Sandig zentrale Bedeutung von Dichtung als Sprachklang und Ich-Stimme hinzu.

\section{Welt- und Selbsterfahrungsspiele: Reiselyrik der Gegenwart, auch eine Literaturbetriebsfrage}

Mit Ulrike Almut Sandigs Dickicht manifestiert sich für die neue deutsche Reiselyrik somit noch eine weitere Ebene, d.h. die von Kolbe und Wagner auf je unterschiedliche Weise heiter und ernst umspielte Frage nach dem ,Gewinn' von Reisen und Reiseliteratur, seitdem die literarische Reihe die Postmoderne erreicht hat und eigentlich nichts mehr erreichbar zu sein scheint, wird noch einmal metaisiert. Wenn sich Reiselyrik nun mit Fremdem (in Welt und Selbst) auseinandersetzt, bekommt die dabei notwendige Suchbewegung für das lyrische und oft auch das Dichter(innen)-Ich eine generellere Bedeutung, denn sie steht als Thematik zugleich dafür, wie Gegenwartslyrik poetisch und poetologisch überhaupt vorgehen kann. Dies meint, dass die eingangs in Erinnerung gerufene Grundfunktion von Literatur, die Entautomatisierung von Wahrnehmung, vermittels des Reisesujets nicht nur genutzt wird, sondern auch als solche in den Blick kommt.

Noch einmal anders perspektivieren lässt sich die ReiselyrikKonjunktur schließlich - hier freilich im bloßen Ausblick -, wenn man sie literatursoziologisch befragt, z.B. mithilfe des eingangs ange- 
sprochenen Themenhefts von Das Gedicht, in dem der Herausgeber trotz genereller Skepsis den Mehrwert realen Reisens in der digital vernetzten Gegenwart feststellt:

Für die Poesie zu verreisen ist eine Moleste [...], die man als Dichter für die gute Sache auf sich nehmen kann. Denn in der Ferne warten vielleicht mir unbekannte Poeten, deren VersKosmen ich kennenlernen kann, wenn ich mich auf eine reale Entdeckungsreise begebe. Und was gibt es Schöneres, als in Begleitung eines aufgeweckten Dichters disputierend durch die Stadt zu ziehen und sich dem fremden Terrain mithilfe des vertrauten Mediums Lyrik anzunähern?40

Reiselyrik erscheint insofern als expliziter Ausweis einer Art Dichterhabitus (sicherlich: nicht nur) der Gegenwart, den etwa auch Jan Wagner in der seiner Lyrik ,beiläufigen Prosa' in Die Sandale des Propheten ansetzt und mit dem er Autorenreisen enthusiastisch bejaht ${ }^{41}$. Dass lyrische (wie andere) Autorinnen und Autoren der Gegenwart aufgrund zahlreicher Einladungen, Stipendien u.ä. de facto viel auf Reisen sein können, was sich z.B. entlang an Uwe Kolbes Lyrikbänden der letzten Jahrzehnte gut belegen ließe, ist insofern nicht nur ,schön für sie', sondern auch eine stete Quelle von Reiselyrik, gewissermaßen aller postmodernen Skepsis seit Brinkmann u.a. zum Trotz.

40. - Anton G. Leitner, ,Reise, Dichter - oder bleib, wo du bist! Ein StubenhockerReport", Das Gedicht, a.a.O., S. 105f., hier S. 106.

41. - Vgl. Jan Wagner, „Finnisches Leuchten“, in: Ders., Die Sandale des Propheten, a.a.O., S. 214-220, hier S. 214. 
